\title{
Oral adsorbent AST-120 ameliorates endothelial dysfunction independent of renal function in rats with subtotal nephrectomy
}

\author{
Tamehachi Namikoshi ${ }^{1}$, Naruya Tomita ${ }^{1}$, Minoru Satoh $^{1}$, Takeo Sakuta ${ }^{1}$, Atsunori Kuwabara ${ }^{1}$, \\ Shinya Kobayashi ${ }^{1}$, Yuusuke Higuchi ${ }^{2}$, Fuyuhiko Nishijima ${ }^{2}$ and Naoki Kashihara ${ }^{1}$
}

It is important to consider a strategy to halt the development of cardiovascular disease (CVD) in patients with chronic kidney disease (CKD). Oral adsorbent AST-120 retards deterioration in renal function, reducing indoxyl sulfate (IS) accumulation. The aim of this study was to determine whether AST-120 improves endothelial dysfunction by reducing oxidative/nitrative stress in a rat-CKD model. Subtotally nephrectomized $(\mathrm{Nx})$ rats aged 17 weeks were divided into two groups: control rats and rats orally treated with AST-120. Two weeks after initiation of AST-120, serum and urinary IS levels, renal histological scores and endothelium-dependent vascular responses (EDVRs) in the aorta were investigated. EDVR in 5-h incubation with $250 \mu \mathrm{gl}^{-1}$ IS was also examined in normal rat aortas. Nitrotyrosine content, mRNA expression of p47phox, a nicotinamide adenine dinucleotide phosphate (NADPH) oxidase component, and expression and phosphorylation (serine-1177) of endothelial nitric oxide synthase (eNOS) in the aorta were examined in untreated and treated Nx rats. At the end of treatment, renal function and histological scores were not different in the two groups. AST-120 prevented the elevation of serum IS level in Nx rats, reducing urinary IS excretion, and ameliorated decreased EDVR in Nx rats. Incubation with IS tended to reduce EDVR in normal aortas, albeit insignificantly. AST-120 also suppressed nitrotyrosine accumulation and inhibited p47phox expression in Nx rats.

The eNOS expression and phosphorylation were similar in the two groups. In conclusion, AST-120 ameliorated endothelial dysfunction and alleviated oxidative/nitrative stress in the aorta through reduced accumulation of IS, independent of renal function, in a rat CKD model.

Hypertension Research (2009) 32, 194-200; doi:10.1038/hr.2008.29; published online 16 January 2009

Keywords: AST-120; CKD; endothelial dysfunction; IS; oxidative/nitrative stress

\section{INTRODUCTION}

Chronic kidney disease (CKD) leading to end-stage renal disease is a risk factor for the development of cardiovascular disease (CVD) and consequently for increased morbidity and mortality associated with this disease. ${ }^{1}$ Several experimental and clinical studies have documented the renovascular protective effects of inhibitors of the reninangiotensin-aldosterone system (RAAS) and their potential usefulness for retarding the development of CKD. ${ }^{2,3}$ However, the use of these inhibitors has not yet resulted in reduced incidence of CKD. ${ }^{4}$ Therefore, it is important to consider another strategy to halt the development of CVD associated with CKD, in addition to the use of RAAS inhibitors.

Several studies have revealed that oral adsorbent AST-120 retards the deterioration of renal function in patients with CKD partly by inhibiting the accumulation of indoxyl sulfate (IS), one of the uremic toxins. $^{5-7}$ AST-120 was also found to delay the progression of renal dysfunction in Japanese type 2 diabetic patients. ${ }^{8}$ Furthermore, experimental studies also showed that AST-120 prevents the progression of renal injury in rats with subtotal nephrectomy and focal glomerulosclerosis. ${ }^{9}, 10$ The renoprotective effects of AST-120 are thought to be mediated by reduced interstitial fibrosis (IF) through downregulation of transforming growth factor- $\beta 1$ and tissue inhibitor of metalloproteinase- 1 in uremic and diabetic rat kidneys. ${ }^{11,12}$

There is some evidence that AST-120 can also retard the development of CVD in patients with CKD. It was reported to reduce carotid intima-media thickness and arterial stiffness in patients with chronic renal failure. ${ }^{13}$ Furthermore, IS directly stimulated the proliferation of rat vascular smooth muscle cells by activating mitogen-activated protein kinase $\mathrm{e}^{14}$ and also induced aortic calcification with expression of osteoblast-specific proteins and aortic wall thickening in hypertensive rats, ${ }^{15}$ suggesting that AST-120 possibly prevents the progression of atherosclerosis associated with CKD by inhibiting IS accumulation. Intriguingly, endothelial dysfunction is seen in patients with CKD and is most likely linked to cardiovascular complications. ${ }^{16}$ However, it

1Division of Nephrology, Department of Internal Medicine, Kawasaki Medical School, Kurashiki, Japan and ${ }^{2}$ Biomedical Research Laboratories, Kureha Co., Ltd, Tokyo, Japan Correspondence: Dr N Tomita, Division of Nephrology, Department of Internal Medicine, Kawasaki Medical School, 577 Matsushima, Kurashiki $701-0192$, Japan. E-mail: tomita@med.kawasaki-m.ac.jp

Received 9 September 2008; revised 12 November 2008; accepted 30 November 2008; published online 16 January 2009 
remains to be determined whether AST-120 improves endothelial dysfunction associated with CKD.

AST-120 was also reported to reduce oxidative stress in the kidneys of rats with subtotal nephrectomy. ${ }^{17}$ In addition, IS induced free radical production in renal proximal tubular cells ${ }^{18}$ and reduced superoxide scavenging activity in the kidneys of normal and uremic rats. ${ }^{19}$ We previously showed that endothelial dysfunction was associated with enhanced vascular oxidative and nitrative stress in rats with obesity and renal injury. ${ }^{20,21}$ The aim of this study was to determine whether AST-120 improves endothelial dysfunction and reduces vascular oxidative/nitrative stress in subtotally nephrectomized rats. We also examined the altered expression and phosphorylation of endothelial nitric oxide synthase (eNOS) in the aorta.

\section{METHODS}

\section{Animal preparation}

The Ethics Review Committee for Animal Experimentation of the Kawasaki Medical School, Kurashiki, Japan, approved the experimental protocol of this study (No. 07-067). Male Wistar rats were purchased from Japan SLC (Hamamatsu, Japan), housed in a temperature- and humidity-controlled room with $12 / 12 \mathrm{~h}$ light/dark cycles and provided free access to water. All rats underwent five-sixths renal ablation using the method described earlier. ${ }^{17,19}$ Eight weeks later, the subtotally nephrectomized rats (age 17 weeks) were divided randomly into two groups ( $n=12$ in each group): the first group was fed a normal diet (MF; Oriental Yeast, Osaka, Japan) (Nx) and the second group was fed a normal diet containing $0.4 \mathrm{~g}$ of AST- 120 per $100 \mathrm{~g}$ body weight per day (Kureha, Tokyo, Japan) (Nx+AST). Age-matched sham-operated rats fed a normal diet (Sham) were used for comparison. Body weight and food intake per day were measured every week. Two weeks after the initiation of AST-120, rats were killed by intraperitoneal injection of sodium pentobarbital (50 $\mathrm{mg} \mathrm{kg}^{-1}$ body weight). Kidneys and aortas were harvested from all animals for histological and biochemical analyses. Abdominal aortas were frozen in liquid nitrogen and stored at $-80^{\circ} \mathrm{C}$, whereas the left kidneys were fixed in $4 \%$ paraformaldehyde and embedded in paraffin.

\section{Physiological and biochemical measurements}

Systolic arterial blood pressure (SBP) was measured by the tail-cuff method in pre-warmed rats (BP-98A; Softron, Tokyo, Japan). ${ }^{22}$ To collect urine samples, rats were placed in metabolic cages for $24 \mathrm{~h}$ and provided tap water but no food. Urinary protein excretion (UPE), serum and urinary creatinine (Cr), serum and urinary IS, and blood urea nitrogen (BUN) levels were measured before and 2 weeks after the initiation of AST-120. Serum and urinary IS were measured by HPLC as described earlier. ${ }^{19}$

\section{Morphological studies}

Four- $\mu \mathrm{m}$-thick sections were prepared from renal tissue samples embedded in paraffin, and stained with periodic acid-Schiff (PAS) and Masson-trichrome (MT). Histological scores were assessed using a Nikon Coolscope (Nikon, Tokyo, Japan). Two investigators semiquantitatively analyzed PAS-stained sections in a blind manner. The severity of glomerular injury was evaluated by the glomerulosclerosis (GS) score as described earlier: ${ }^{21,23} 0$, no sclerosis; 1 , sclerotic changes in $<25 \%$ of the total area of the glomerulus; 2 , from 25 to $50 \%$ of the total area; 3 , from 50 to $75 \%$ of the total area; 4 , in more than $75 \%$ of the total area. At least 30 glomeruli were randomly selected in each rat and the mean score was calculated. The severity of tubulointerstitial injury was evaluated by the interstitial fibrosis score as described earlier. ${ }^{21,23}$ The fibrotic areas stained blue by MT were measured quantitatively using a color image analyzer (Win ROOF; Mitani, Fukui, Japan). The percentage of interstitial fibrotic areas per cortical field (magnification, $\times 100$ ) was calculated, and the mean percentage in five randomly selected fields was determined as the IF score for each rat.

\section{Endothelium-dependent vascular responses}

Endothelium-dependent vascular responses (EDVR) were measured after the preparation of thoracic rat aortic rings as described earlier. ${ }^{20,21}$ Briefly, cylindrical 3-mm-long segments were cut from the aortas and bathed in $5 \mathrm{ml}$ of Krebs bicarbonate saline equilibrated with $95 \% \mathrm{O}_{2}$ and $5 \% \mathrm{CO}_{2}$, and maintained at $37^{\circ} \mathrm{C}$. Aortic segments were suspended under $1 \mathrm{~g}$ tension, depolarized with $50 \mathrm{mmoll}^{-1}$ potassium chloride and preconstricted by adding $3 \times 10^{-7} \mathrm{moll}^{-1}$ norepinephrine. After the contraction force reached a plateau, acetylcholine, an endothelium-dependent vasodilator, was added in incremental concentrations $\left(10^{-9}-10^{-5} \mathrm{moll}^{-1}\right)$ to the bath. The force of isometric contraction was measured using a force-displacement transducer (Model MTOB-1Z; Labo Support, Osaka, Japan). Responses to acetylcholine were expressed as a percentage of the precontracted tension induced by norepinephrine. To validate IS-induced endothelial dysfunction, thoracic aortic rings obtained from male Wistar rats aged 10 weeks were incubated in Dulbecco's modified eagle's medium (DMEM) with or without $250 \mu \mathrm{g} \mathrm{ml}^{-1}$ IS (SigmaAldrich, Tokyo, Japan), containing $120 \mathrm{U} \mathrm{ml}^{-1}$ penicillin, $120 \mu \mathrm{g} \mathrm{ml}^{-1}$ streptomycin, $50 \mu \mathrm{g} \mathrm{ml}^{-1}$ polymyxin B and $1 \%$ fetal bovine serum, for $5 \mathrm{~h}$ at $37^{\circ} \mathrm{C}$ $(n=4)$, as described earlier. ${ }^{24,25}$ The IS concentration and incubation time accepted in this study were the maximum of those described in earlier in vitro studies. ${ }^{26,27}$ Vascular responses were then examined.

\section{Immunohistochemistry}

Four- $\mu \mathrm{m}$-thick sections were prepared from aortic tissue samples embedded in paraffin. Immunohistochemical staining and evaluation for nitrotyrosine, a tracer of nitrative stress, was performed in aorta as reported earlier. ${ }^{28,29} \mathrm{~A}$ rabbit anti-nitrotyrosine polyclonal antibody $\left(5 \mu \mathrm{g} \mathrm{ml}^{-1}\right.$ ) (Upstate Biotechnology,

Table 1 Changes in various physiological and biochemical parameters in sham-operated (Sham), subtotally nephrectomized (Nx) and subtotally nephrectomized-AST-120-treated rats (Nx+AST)

\begin{tabular}{|c|c|c|c|}
\hline & Sham & $N x$ & $N x+A S T$ \\
\hline \multicolumn{4}{|l|}{ Body weight, $g$} \\
\hline Baseline & $328 \pm 5$ & $293 \pm 3^{*}$ & $296 \pm 3^{*}$ \\
\hline 2-week AST-120 & $316 \pm 5$ & $279 \pm 3^{* *}$ & $281 \pm 4^{* *}$ \\
\hline \multicolumn{4}{|l|}{ Food intake, gday ${ }^{-1}$} \\
\hline Baseline & $17.7 \pm 0.4$ & $14.1 \pm 0.4^{*}$ & $15.1 \pm 0.3^{*}$ \\
\hline 2-week AST-120 & $17.5 \pm 0.3$ & $14.6 \pm 0.3^{* *}$ & $15.4 \pm 0.3^{* *}$ \\
\hline \multicolumn{4}{|c|}{ Systolic blood pressure, $\mathrm{mm} \mathrm{Hg}$} \\
\hline Baseline & $122 \pm 2$ & $146 \pm 5^{*}$ & $143 \pm 4^{*}$ \\
\hline 2-week AST-120 & $132 \pm 4$ & $160 \pm 5^{* *}$ & $151 \pm 5^{* *}$ \\
\hline \multicolumn{4}{|c|}{ Blood urea nitrogen, $\mathrm{mg}$ per $100 \mathrm{ml}$} \\
\hline Baseline & $16 \pm 1$ & $36 \pm 3^{*}$ & $35 \pm 2^{*}$ \\
\hline 2-week AST-120 & $18 \pm 1$ & $44 \pm 4^{* *}$ & $41 \pm 4^{* *}$ \\
\hline
\end{tabular}

Serum creatinine, $\mathrm{mg}$ per $100 \mathrm{ml}$

$\begin{array}{llll}\text { Baseline } & 0.64 \pm 0.03 & 1.03 \pm 0.05^{*} & 1.03 \pm 0.05^{*} \\ \text { 2-week AST-120 } & 0.52 \pm 0.04 & 1.08 \pm 0.10^{* *} & 1.04 \pm 0.08^{* *}\end{array}$

$\begin{array}{cccc}\text { Ccr, } m / \text { per min per 100 g body weight } & & \\ \text { Baseline } & 0.39 \pm 0.03 & 0.23 \pm 0.01^{*} & 0.23 \pm 0.01^{*} \\ \text { 2-week AST-120 } & 0.48 \pm 0.03 & 0.27 \pm 0.02^{* *} & 0.28 \pm 0.03^{* *}\end{array}$

Urinary protein excretion, mgday $^{-1}$

$\begin{array}{llcc}\text { Baseline } & 9.0 \pm 0.3 & 55.2 \pm 11.1^{*} & 51.3 \pm 9.6^{*} \\ \text { 2-week AST-120 } & 9.3 \pm 0.7 & 157.6 \pm 30.7^{* *} & 123.2 \pm 19.7^{* *}\end{array}$

Ccr, creatinine clearance. Data are the mean \pm s.e.m. of 12 rats in each group. ${ }^{*} P<0.01$ vs. the Sham group at baseline ( 8 weeks after subtotal nephrectomy), ${ }^{* *} P<0.01$ vs. the Sham group 2 weeks after the initiation of AST-120. 

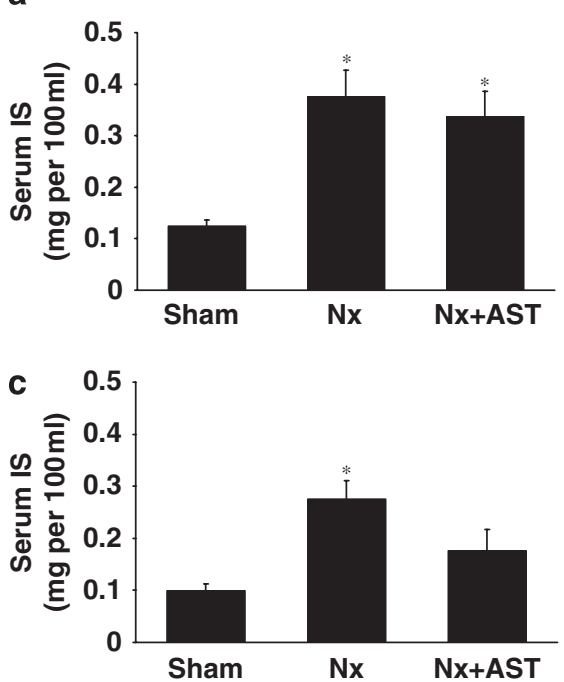

b
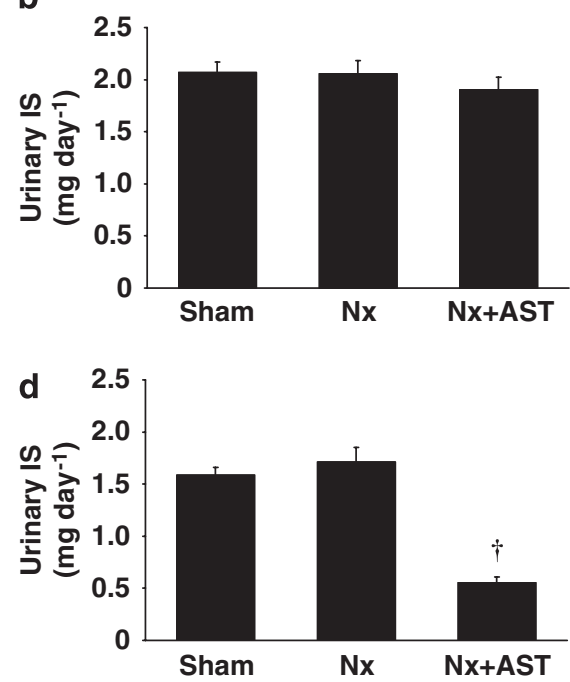

Figure 1 Serum and urinary indoxyl sulphate (IS) levels before and 2 weeks after the initiation of AST-120. (a) Before the initiation of AST-120, serum IS increased significantly in subtotally nephrectomized rats $(\mathrm{Nx})$ and rats treated with AST-120 (Nx+AST) compared with sham-operated rats (Sham). (b) Urinary IS was not significantly different in the three groups. (c) At 2 weeks after the initiation of AST-120, serum IS increased significantly in the Nx group, but not in the $\mathrm{Nx}+\mathrm{AST}$ group, compared with the Sham group. (d) Urinary IS decreased significantly in the $\mathrm{Nx}+\mathrm{AST}$ group compared with both the Sham and Nx groups. Data are the mean \pm s.e.m. of 12 rats in each group. ${ }^{*} P<0.01$ vs. the Sham group, ${ }^{\dagger} P<0.01$ vs. the Sham and Nx groups.

Lake Placid, NY, USA) was used as the primary antibody. Antibody binding was detected using a Histofine Simple Stain MAX-PO (MULTI) Kit (Nichirei, Tokyo, Japan) and 3,3'-diaminobenzidine (Sigma-Aldrich, Tokyo, Japan), counterstained by hematoxylin. Stained sections were photographed using the Nikon Eclipse E800 Microscope (Nikon) and digitized into $1280 \times 1024$ pixel colored images using the Nikon ACT-1 DXM 1200 software (Nikon). The positive areas were measured quantitatively using a color image analyzer (Win ROOF). The percentage of positive areas per aortic field containing endothelial and smooth muscle layers (magnification, $\times 400$ ) was calculated, and the mean percentage in four randomly selected fields was determined as the net score for each rat.

\section{Real-time quantitative PCR}

RNA isolation and real-time quantitative PCR were performed as described earlier. ${ }^{21,30}$ Briefly, total RNA was extracted from the aorta with TRIzol (Invitrogen, Tokyo, Japan). Reverse transcriptase reactions were performed using a Ready-To-Go T-Primed First-Strand Kit (Amersham Biosciences, Piscataway, NJ, USA). Real-time quantitative PCR was performed on an ABI Prism 7700 sequence-detection system (Applied Biosystems, Foster City, CA, USA). Primers and probes used for the p47phox, which is one among the nicotinamide adenine dinucleotide phosphate (NADPH) oxidase components, were $5^{\prime}$-GTGAAGCCATCGAGGTCATTC- ${ }^{\prime}$ as forward, 5'-CCCGCGG CTTCTAATCTGT-3' as reverse and $5^{\prime}$-FAM-CATCACCGGCTACTTCCCATC CATGT-TAMRA- $3^{\prime}$ as TaqMan probe. Changes in expression were quantified by standardization against RNA levels corrected for glyceraldehyde-3-phosphate dehydrogenase expression in the sample.

\section{Western blot analysis}

Western blotting was performed to detect eNOS and phosphorylation of eNOS at serine residue 1177 (p-eNOS) in aortas, as described earlier. ${ }^{28}$ At protein extraction, complete EDTA-free and PhosSTOP (Roche Applied Science, Mannheim, Germany) were used for inhibition of protease and phosphatase, respectively. Extracted proteins (100 $\mu \mathrm{g}$ per lane) were resolved by $7.5 \%$ SDSPAGE and then electrotransferred onto polyvinylidene difluoride membranes (Millipore, Bedford, MA, USA). Membranes were blocked and incubated with rabbit anti-eNOS, rabbit anti-p-eNOS or goat anti- $\alpha$-tubulin polyclonal antibodies (Santa Cruz Biotechnology, Santa Cruz, CA, USA). After washing, they were incubated with horseradish peroxidase-conjugated donkey anti-rabbit IgG or the anti-goat IgG (1:5000; Santa Cruz Biotechnology). The resolved proteins were visualized using an enhanced chemiluminescence method (ECL Plus; Amersham Biosciences). The relative optical densities of bands were quantified using NIH Image analysis software V1.61 (National Institutes of Health, Bethesda, MD, USA).

\section{Statistical analysis}

The values are expressed as mean \pm s.e.m. All parameters were evaluated with the two-tailed unpaired Welch's $t$-test or Mann-Whitney's $U$-test, or compared by one-way analysis of variance when multiple mean comparisons were required. A $P$-value $<0.05$ was considered to be statistically significant.

\section{RESULTS}

Physiological and biochemical parameters

Table 1 summarizes the physiological and biochemical data at baseline (8 weeks after subtotal nephrectomy, and before the initiation of AST-120). Body weight, food intake and creatinine clearance (Ccr) were significantly lower, and SBP, BUN, serum Cr and UPE were significantly higher in the $\mathrm{Nx}$ and $\mathrm{Nx}+\mathrm{AST}$ groups compared with the Sham group $(P<0.01)$. None of the parameters were significantly different between the $\mathrm{Nx}$ and $\mathrm{Nx}+\mathrm{AST}$ groups at baseline. Table 1 also depicts the physiological and biochemical data 2 weeks after the initiation of AST-120. Body weight, food intake and Ccr were significantly lower, and SBP, BUN, serum Cr and UPE were significantly higher in the $\mathrm{Nx}$ and $\mathrm{Nx}+\mathrm{AST}$ groups compared with the Sham group $(P<0.01)$. Furthermore, SBP and UPE tended to be lower in the $\mathrm{Nx}+\mathrm{AST}$ group than in the $\mathrm{Nx}$ group, albeit insignificantly. BUN, serum $\mathrm{Cr}$ and $\mathrm{Ccr}$ were not different in the $\mathrm{Nx}+\mathrm{AST}$ group compared with the Nx group. These results indicate that the administration of AST-120 for 2 weeks did not significantly change the renal function in this model.

\section{Serum and urinary IS levels}

At baseline, serum IS was similar in the $\mathrm{Nx}(0.38 \pm 0.05 \mathrm{mg}$ per $100 \mathrm{ml})$ and $\mathrm{Nx}+\mathrm{AST}(0.34 \pm 0.05 \mathrm{mg}$ per $100 \mathrm{ml})$ groups, but the values of both groups were significantly higher than those of the Sham 
group $(0.12 \pm 0.01 \mathrm{mg}$ per $100 \mathrm{ml} ; P<0.01$ vs. Sham) (Figure 1a). Urinary IS was not significantly different in the three groups (Sham: $2.07 \pm 0.10 \mathrm{mg} \mathrm{day}^{-1}, \mathrm{Nx}: 2.06 \pm 0.12 \mathrm{mg} \mathrm{day}^{-1}, \mathrm{Nx}+\mathrm{AST}$ : $1.90 \pm 0.12 \mathrm{mg} \mathrm{day}^{-1}$, Figure 1b). At 2 weeks after the initiation of AST-120, serum IS increased significantly in the $\mathrm{Nx}$ group $(0.28 \pm 0.04 \mathrm{mg}$ per $100 \mathrm{ml})$ but not in the $\mathrm{Nx}+\mathrm{AST}$ group $(0.18 \pm 0.04 \mathrm{mg}$ per $100 \mathrm{ml})$ compared with the Sham group $(0.10 \pm 0.01 \mathrm{mg}$ per $100 \mathrm{ml}, P<0.01$ vs. Sham) (Figure 1c). It was lower in the $\mathrm{Nx}+\mathrm{AST}$ group than in the $\mathrm{Nx}$ group, albeit insignificantly $(P=0.083)$. Urinary IS tended to be higher in the $\mathrm{Nx}$ group $\left(1.71 \pm 0.14 \mathrm{mg} \mathrm{day}^{-1}\right)$ than in the Sham group $\left(1.59 \pm 0.07 \mathrm{mg} \mathrm{day}^{-1}\right)$, albeit insignificantly (Figure $1 \mathrm{~d}$ ), but was significantly lower in the $\mathrm{Nx}+\mathrm{AST}$ group $\left(0.56 \pm 0.05 \mathrm{mg} \mathrm{day}^{-1}\right)$ than in the Sham and Nx groups $(P<0.01)$. These results indicate that AST-120 prevented the increase in serum IS level and decreased the urinary IS level in this model.

\section{Morphological studies}

The GS and IF scores in renal tissues 2 weeks after the initiation of AST-120 were both significantly higher in the $\mathrm{Nx}$ and $\mathrm{Nx}+$ AST groups than in the Sham group (Table 2). Both scores tended to be lower in the $\mathrm{Nx}+\mathrm{AST}$ group than in the $\mathrm{Nx}$ group, albeit insignificantly. These results suggest that a 2 -week treatment with AST-120 did not change the severity of renal damage in this model.

Endothelium-dependent vascular responses

At $10^{-8}$ and $10^{-5} \mathrm{moll}^{-1}$ acetylcholine, the relaxation responses in the $\mathrm{Nx}$ group were significantly lower than in the Sham group $(P<0.05)$ (Figure 2a). Administration of AST-120 for 2 weeks significantly improved the relaxation response in the $\mathrm{Nx}$ group at $10^{-7}$ $10^{-5} \mathrm{moll}^{-1}$ acetylcholine $(P<0.05)$. It is to be noted that the relaxation response at $10^{-5} \mathrm{moll}^{-1}$ acetylcholine correlated significantly with the serum IS level $(P=0.011)$ (Figure $2 \mathrm{~b})$. In addition, a 5 -h incubation with $250 \mu \mathrm{g} \mathrm{ml}^{-1}$ IS tended to reduce the acetylcholine-induced relaxation response in normal aortas, albeit insignificantly $(P=0.065)$ (Figure 2c). These results indicate that AST-120 ameliorates the decreased endothelium-dependent vascular response independent of the renal function in this model, and that the ameliorative effect of AST-120 on endothelial dysfunction was associated with decreased level of serum IS.

\section{Immunohistochemistry}

The Sham aorta showed weak staining for nitrotyrosine in endothelial and smooth muscle cells (Figure 3a). In the $\mathrm{Nx}$ aorta, enhanced staining was observed in endothelial cells (arrowheads) and smooth muscle cells compared with the Sham aorta (Figure 3b). Staining was weaker in the $\mathrm{Nx}+\mathrm{AST}$ aorta compared with the $\mathrm{Nx}$ aorta (Figure 3c). Figure $3 \mathrm{~d}$ shows the results of quantitative analysis of nitrotyrosine staining. The area stained for nitrotyrosine was significantly larger in the $\mathrm{Nx}$ group, but not in the $\mathrm{Nx}+\mathrm{AST}$ group, compared with the Sham group. Furthermore, the area tended to be smaller in the

Table 2 Renal histological scores at the end of this study

\begin{tabular}{lccc}
\hline & Sham & $N x$ & $N x+A S T$ \\
\hline Glomerulosclerosis score & $0.18 \pm 0.02$ & $1.33 \pm 0.14^{*}$ & $1.23 \pm 0.14^{*}$ \\
Interstitial fibrosis score (\%) & $4.6 \pm 0.5$ & $16.9 \pm 2.2^{*}$ & $13.8 \pm 1.5^{*}$ \\
\hline Nx, nephrectomized rats; Nx+AST, subtotally nephrectomized-AST-120-treated rats. \\
$\begin{array}{l}\text { Data are mean } \pm \text { s.e.m. of 10 rats in each group. } \\
{ }^{*} P<0.01 \text { vs. the Sham group. }\end{array}$
\end{tabular}

$\mathrm{Nx}+$ AST group than in the $\mathrm{Nx}$ group, albeit insignificantly. These results indicate that AST-120 suppresses nitrotyrosine accumulation in the aorta independent of renal function in this model.

\section{Real-time quantitative PCR}

One of the major sources of superoxide $\left(\mathrm{O}_{2}{ }^{-}\right)$production in the blood vessels is NADPH oxidase. ${ }^{31}$ We examined the mRNA expression of p47phox, one of the NADPH oxidase components, in the aorta using real-time PCR. As shown in Figure 4, the mRNA expression of

a
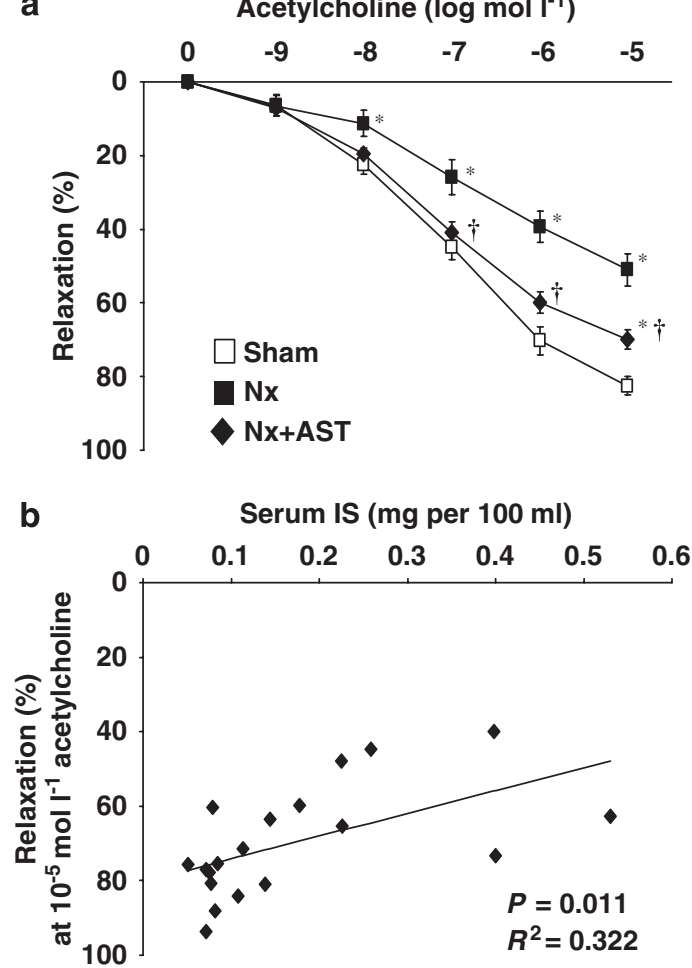

C

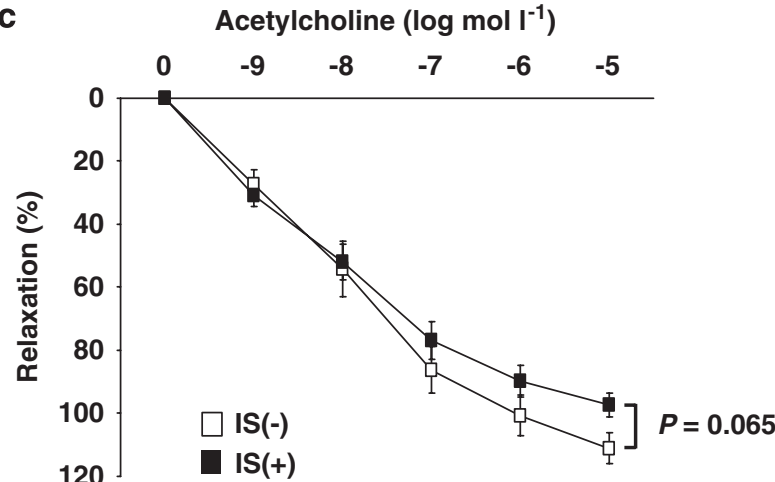

Figure 2 Endothelium-dependent vascular response (EDVR) in thoracic aorta. (a) The relaxation response in subtotally nephrectomized rats ( $\mathrm{Nx})$ was significantly lower than that in sham-operated rats (Sham), at $10^{-8}$ and $10^{-5} \mathrm{moll}^{-1}$ acetylcholine. The relaxation response in subtotally nephrectomized rats treated with AST-120 ( $\mathrm{Nx}+\mathrm{AST})$ was significantly improved compared with the $\mathrm{Nx}$ group at $10^{-7}$ to $10^{-5} \mathrm{moll} \mathrm{I}^{-1}$ acetylcholine. (b) The relaxation response at $10^{-5} \mathrm{moll}^{-1}$ acetylcholine correlated significantly with serum indoxyl sulfate (IS) level. (c) In addition, 5-h incubation with $250 \mu \mathrm{g} \mathrm{ml}^{-1}$ IS reduced acetylcholine-induced relaxation response in normal aortas, albeit insignificantly. Data are the mean \pm s.e.m. of $4-7$ rats in each group. ${ }^{*} P<0.05$ vs. the Sham group, $\uparrow P<0.05$ vs. the Nx group. 

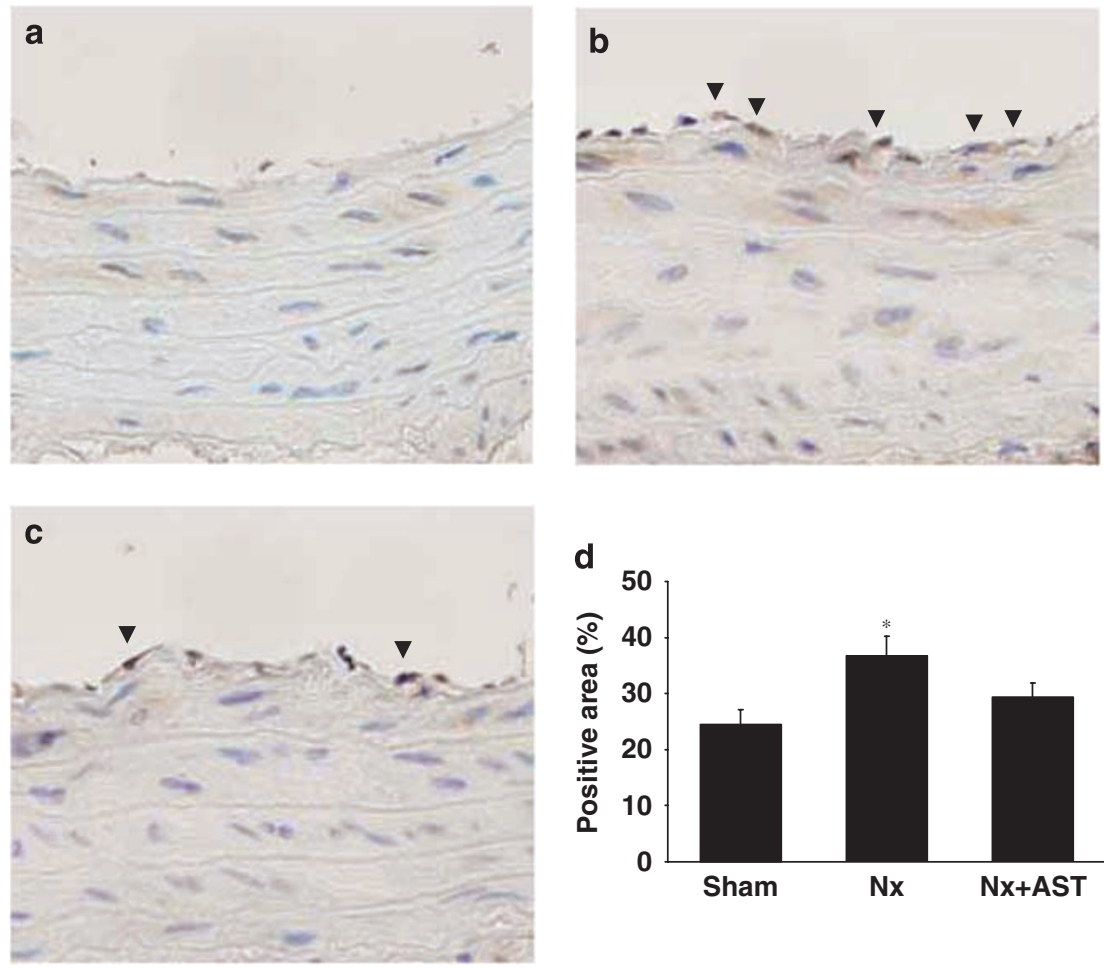

Figure $3 \mathrm{Immunohistochemical} \mathrm{photomicrographs} \mathrm{depicting} \mathrm{nitrotyrosine} \mathrm{accumulation} \mathrm{in} \mathrm{the} \mathrm{aorta} \mathrm{(a-c)} \mathrm{and} \mathrm{the} \mathrm{results} \mathrm{of} \mathrm{quantitative} \mathrm{assessment} \mathrm{of} \mathrm{the}$ area positive for nitrotyrosine (d). (a) The aorta of a representative sham-operated rat (Sham) showed weak staining in endothelial and smooth muscle cells. (b) In the aorta of a subtotally nephrectomized rat ( $\mathrm{Nx}$ ), strong staining was observed in endothelial cells (arrowheads) and smooth muscle cells compared with the Sham aorta. (c) Staining for nitrotyrosine was weak in the aorta of a subtotally nephrectomized rat treated with AST-120 (Nx+AST) compared with the Nx aorta. (d) The nitrotyrosine-stained area was significantly larger in the Nx group but not in the Nx+AST group, compared with the Sham group. The area tended to be smaller in the $\mathrm{Nx}+\mathrm{AST}$ group than in the $\mathrm{Nx}$ group, albeit insignificantly. Magnification, $\times 400$. Data are the mean \pm s.e.m. of 9 or 10 rats in each group. ${ }^{*} P<0.05$ vs. the Sham group.

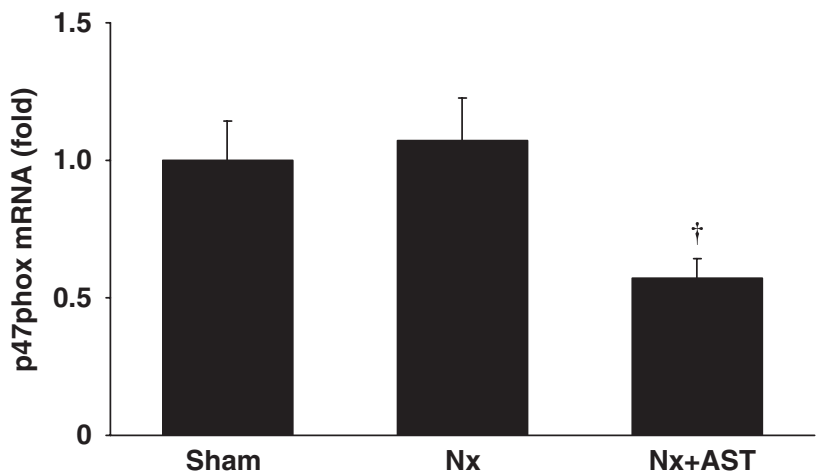

Figure 4 Real-time PCR analysis for mRNA expression of p47phox, a nicotinamide adenine dinucleotide phosphate (NADPH) oxidase component, in aortas. The mRNA expression levels of p47phox were similar in shamoperated rats (Sham) and subtotally nephrectomized rats $(\mathrm{Nx})$. The level was significantly decreased in subtotally nephrectomized rats treated with AST-120 ( $\mathrm{Nx}+\mathrm{AST})$ compared with the Sham and Nx groups. Data are the mean \pm s.e.m. of 7 or 8 rats. ${ }^{\dagger} P<0.05$ vs. the Sham and $\mathrm{Nx}$ groups.

p47phox was similar in the Sham and Nx groups. However, it was significantly lower in the $\mathrm{Nx}+\mathrm{AST}$ group than in the Sham and $\mathrm{Nx}$ groups $(P<0.05)$. These results suggest that the ameliorative effects of AST-120 on endothelial dysfunction and nitrotyrosine accumulation in the aorta are mediated through downregulation of a component of NADPH oxidase.

\section{Western blot analysis}

Endothelial dysfunction is possibly caused by decreased NO production through inhibition of expression and/or phosphorylation of eNOS. To test this, western blotting for eNOS and p-eNOS in aortas was performed. Bands for eNOS and p-eNOS were detected at $135 \mathrm{kDa}$. The protein expressions of eNOS and p-eNOS were evaluated as the eNOS/ $\alpha$-tubulin ratio (Figure $5 \mathrm{a}$ ) and p-eNOS/eNOS ratio (Figure 5b), respectively. Both expressions were similar in the three groups. These results indicate that expressions of eNOS and p-eNOS were not different in the Nx group compared with the Sham group, and that AST-120 did not modify their expressions.

\section{DISCUSSION}

In this study, we showed that AST-120 improved the reduced endothelium-dependent vascular response of the aorta in subtotally nephrectomized rats by reducing the serum and urinary IS levels. The endothelium-dependent vascular response of the aorta at $10^{-5} \mathrm{moll}^{-1}$ acetylcholine correlated significantly with the serum IS level, and incubation with IS tended to reduce the endothelium-dependent vascular response of normal aortas. AST-120 also prevented the accumulation of nitrotyrosine in the vascular wall in this model, inhibiting the mRNA expression of p47phox, one of the NADPH oxidase components, although it did not modify eNOS expression or phosphorylation at serine residue 1177 . Importantly, these effects of AST-120 were independent of renal function. Considered together, the results suggest that AST-120 treatment of rats with subtotal nephrectomy resulted in amelioration of vascular endothelial dysfunc- 
a
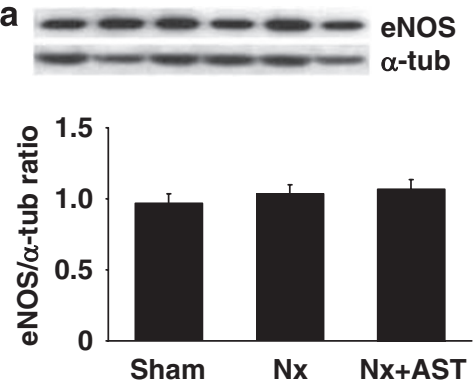

b
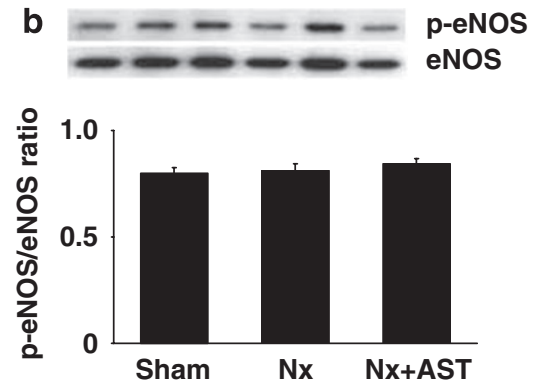

Figure 5 Western blot analysis for endothelial nitric oxide (eNOS) and phosphorylation of eNOS at serine residue 1177 (p-eNOS) in aortas. Bands for eNOS and $\mathrm{p}$-eNOS were detected at $135 \mathrm{kDa}$. The protein expression levels of eNOS and p-eNOS were evaluated as eNOS/ $\alpha$-tubulin ( $\alpha$-tub) ratio (a) and $\mathrm{p}$-eNOS/ eNOS ratio (b), respectively. The expression levels were similar in sham-operated rats (Sham), subtotally nephrectomized rats (Nx) and subtotally nephrectomized rats treated with AST-120 (Nx+AST).

tion and alleviated oxidative/nitrative stress in the vasculature by reducing IS accumulation, independent of renal function.

One of the pathogenetic mechanisms of endothelial dysfunction is oxidative/nitrative stress. ${ }^{21,29}$ Enhanced $\mathrm{O}_{2}{ }^{-}$production in the vasculature reduces $\mathrm{NO}$ bioavailability through scavenging $\mathrm{NO}$ by $\mathrm{O}_{2}{ }^{-}$, followed by endothelial dysfunction. ${ }^{31}$ Peroxynitrite is generated by the immediate reaction of $\mathrm{NO}$ and $\mathrm{O}_{2}{ }^{-}$and is considered cytotoxic as it modifies tyrosine residues in proteins to generate nitrotyrosine. ${ }^{32}$ In this regard, ebselen, a peroxynitrite scavenger, was reported to reduce nitrotyrosine accumulation in the aorta and ameliorate the acetylcholine-induced vasorelaxation in diabetic Zucker rats. ${ }^{29}$ Therefore, the reduced endothelium-dependent vascular response of the aorta in the $\mathrm{Nx}$ group of this study may be caused by enhanced oxidative/nitrative stress, as nitrotyrosine accumulation in the aorta was increased significantly in the $\mathrm{Nx}$ group compared with the Sham group. AST-120 improved the decreased endothelium-dependent vascular response in the aorta and concomitantly prevented the accumulation of nitrotyrosine in the aorta of $\mathrm{Nx}$ rats. Taken together, we consider that IS accumulation in the CKD status enhanced $\mathrm{O}_{2}{ }^{-}$production in the vasculature, that $\mathrm{O}_{2}^{-}$immediately reacted with $\mathrm{NO}$ to form peroxynitrite, and that nitrotyrosine accumulation was then augmented in vasculature.

Earlier in vitro studies have reported that IS can induce endothelial damage. $^{33,34}$ IS inhibited endothelial proliferation and wound repair, and increased endothelial microparticles in human umbilical vein endothelial cells (HUVEC). In this study, we showed that the endothelium-dependent aortic response correlated significantly with serum IS levels. In addition, incubation with IS tended to reduce the endothelium-dependent vascular response of normal aortas in the ex vivo study, albeit insignificantly. These findings suggest that accumulation of IS causes endothelial dysfunction. We accepted $250 \mu \mathrm{g} \mathrm{ml}^{-1}$ IS concentration in this ex vivo study as it was the maximum of those described in earlier in vitro studies. An earlier study reported that the average plasma IS level was $35.2 \mu \mathrm{g} \mathrm{ml}^{-1}$, ranging from 1.8 to $86.2 \mu \mathrm{g} \mathrm{ml}^{-1}$, with a median of $33.3 \mu \mathrm{g} \mathrm{ml}^{-1}$ in Japanese hemodialysis patients. ${ }^{35}$ The ex vivo EDVR with or without $50 \mu \mathrm{g} \mathrm{ml}^{-1}$ IS was also investigated in our study; however, the EDVR with $50 \mu \mathrm{g} \mathrm{ml}^{-1}$ IS was not significantly different from that without IS (data not shown). It may be because of the difference between in vivo and ex vivo experiments. In addition, other uremic toxins may play a role in AST-120-mediated improvement of endothelial dysfunction.

Recent studies showed that IS enhanced reactive oxygen species production in HUVEC, increasing NADPH oxidase activity and decreasing glutathione levels. ${ }^{26,27}$ Therefore, IS accumulation may enhance $\mathrm{O}_{2}{ }^{-}$production and oxidative/nitrative stress in vascular endothelial cells, followed by endothelial dysfunction. We investigated the mRNA expression of p47phox, one of the NADPH oxidase components, in the aorta to elucidate the pathogenic mechanism of $\mathrm{O}_{2}^{-}$production by IS. The results showed that AST-120 significantly reduced $\mathrm{p} 47$ phox expression in the Nx group. However, we could not assess the mRNA expression levels of other components of NADPH oxidase in this study because of their extremely low expression levels. It remains to be determined whether enhanced $\mathrm{O}_{2}{ }^{-}$production by IS in the vasculature is dependent on NADPH oxidase or other mechanisms (e.g., superoxide dismutase, glutathione).

In this study, we examined the expression and phosphorylation of eNOS in the aorta, as endothelial function is dependent on the expression and phosphorylation of eNOS. The results showed no changes in both the expression and phosphorylation of eNOS in the aorta in the Nx group compared with the Sham group, and AST-120 did not modify these expressions. The results indicate that the altered endothelium-dependent vascular response of the aorta was not related to the expression and phosphorylation of eNOS in our rats. Therefore, we consider that reduced NO bioavailability induced endothelial dysfunction in the $\mathrm{Nx}$ group through scavenging $\mathrm{NO}$ by $\mathrm{O}_{2}{ }^{-}$, but not eNOS dysfunction, in this study.

It is important to note that AST-120 ameliorated the reduced vascular response in the $\mathrm{Nx}$ group, independent of renal function. Ccr and renal histological scores were not significantly different between the $\mathrm{Nx}$ and $\mathrm{Nx}+\mathrm{AST}$ groups because of the short duration of AST-120 treatment. AST-120 probably ameliorates endothelial dysfunction by reducing IS accumulation, independent of renal function. Clinically, AST-120 may retard the development of CVD in patients with $\mathrm{CKD}$, as it is reported to reduce the intima-media thickness and arterial stiffness of the carotid artery, which are associated with CVD, in patients with CKD. ${ }^{13}$

The limitation of this study is its inability to demonstrate the specificity of the toxicity of IS in the vasculature sufficiently. However, some in vitro studies have revealed the toxicity of IS in vascular cells. ${ }^{14,33,34}$ In addition, oxidative stress induced by IS may damage vascular cells in an indirect manner. ${ }^{26,27,36}$ Therefore, IS accumulation is considered to be one of the pathogenic mechanisms causing endothelial dysfunction in patients with CKD.

In conclusion, the oral adsorbent AST-120 ameliorated endothelial dysfunction by reducing IS accumulation, independent of renal function, in a rat model of CKD. AST-120 also alleviated oxidative/ nitrative stress in the aorta. Therefore, the ameliorative effect of AST120 on endothelial function may be associated with reduced oxidative/ nitrative stress in the vessel wall. Our results suggest that AST-120 could be potentially useful in retarding the development of CVD in 
patients with CKD. Further studies are needed to elucidate the pathogenetic mechanism of uremic toxin-induced endothelial dysfunction.

\section{ACKNOWLEDGEMENTS}

We thank Ms Satomi Hanada, Ms Yoshiko Shirakiya and Ms Yuki Hamada for their excellent technical assistance. This work was supported by a Grant-in-Aid for Young Scientists (Start-up) from the Japan Society for the Promotion of Science (No. 20890271), and by a Research Project Grant from Kawasaki Medical School (No. 20-3030).

1 Go AS, Chertow GM, Fan D, McCulloch CE, Hsu CY. Chronic kidney disease and the risks of death, cardiovascular events, and hospitalization. N Engl J Med 2004; 351: 1296-1305.

2 Ruster C, Wolf G. Renin-angiotensin-aldosterone system and progression of renal disease. J Am Soc Nephrol 2006; 17: 2985-2991.

3 Karalliedde J, Viberti G. Evidence for renoprotection by blockade of the renin-angiotensin-aldosterone system in hypertension and diabetes. J Hum Hypertens 2006; 20: 239-253.

4 Kiberd B. The chronic kidney disease epidemic: stepping back and looking forward. J Am Soc Nephrol 2006; 17: 2967-2973.

5 Niwa T, Nomura T, Sugiyama S, Miyazaki T, Tsukushi S, Tsutsui S. The protein metabolite hypothesis, a model for the progression of renal failure: an oral adsorbent lowers indoxyl sulfate levels in undialyzed uremic patients. Kidney Int (Suppl) 1997; 52: S23-S28.

6 Owada A, Nakao M, Koike J, Ujiie K, Tomita K, Shiigai T. Effects of oral adsorbent AST-120 on the progression of chronic renal failure: a randomized controlled study. Kidney Int (Suppl) 1997; 52: S188-S190.

7 Shoji T, Wada A, Inoue K, Hayashi D, Tomida K, Furumatsu Y, Kaneko T, Okada N, Fukuhara Y, Imai E, Tsubakihara Y. Prospective randomized study evaluating the efficacy of the spherical adsorptive carbon AST-120 in chronic kidney disease patients with moderate decrease in renal function. Nephron Clin Pract 2007; 105: c99-c107.

8 Shimizu H, Okada S, Shinsuke OI, Mori M. Kremezin (AST-120) delays the progression of diabetic nephropathy in Japanese type 2 diabetic patients. Diabetes Care 2005; 28: 2590.

9 Kobayashi N, Maeda A, Horikoshi S, Shirato I, Tomino Y, Ise M. Effects of oral adsorbent AST-120 (Kremezin) on renal function and glomerular injury in early-stage renal failure of subtotal nephrectomized rats. Nephron 2002; 91: 480-485.

10 Sato T, Liang K, Vaziri ND. Protein restriction and AST-120 improve lipoprotein lipase and VLDL receptor in focal glomerulosclerosis. Kidney Int 2003; 64: 1780-1786.

11 Miyazaki T, Aoyama I, Ise M, Seo H, Niwa T. An oral sorbent reduces overload of indoxyl sulphate and gene expression of TGF- $\beta 1$ in uraemic rat kidneys. Nephrol Dial Transplant 2000; 15: 1773-1781.

12 Aoyama I, Shimokata K, Niwa T. Oral adsorbent AST-120 ameliorates interstitial fibrosis and transforming growth factor- $\beta_{1}$ expression in spontaneously diabetic (OLETF) rats. Am J Nephrol 2000; 20: 232-241.

13 Nakamura T, Kawagoe Y, Matsuda T, Ueda Y, Shimada N, Ebihara I, Koide H. Oral adsorbent AST-120 decreases carotid intima-media thickness and arterial stiffness in patients with chronic renal failure. Kidney Blood Press Res 2004; 27: 121-126.

14 Yamamoto H, Tsuruoka S, loka T, Ando H, Ito C, Akimoto T, Fujimura A, Asano Y, Kusano E. Indoxyl sulfate stimulates proliferation of rat vascular smooth muscle cells. Kidney Int 2006; 69: 1780-1785.

15 Adijiang A, Goto S, Uramoto S, Nishijima F, Niwa T. Indoxyl sulphate promotes aortic calcification with expression of osteoblast-specific proteins in hypertensive rats. Nephrol Dial Transplant 2008; 23: 1892-1901.
16 Stam F, van Guldener C, Becker A, Dekker JM, Heine RJ, Bouter LM, Stehouwer CD. Endothelial dysfunction contributes to renal function-associated cardiovascular mortality in a population with mild renal insufficiency: the Hoorn study. J Am Soc Nephrol 2006; 17: 537-545.

17 Nakagawa N, Hasebe N, Sumitomo K, Fujino T, Fukuzawa J, Hirayama T, Kikuchi K. An oral adsorbent, AST-120, suppresses oxidative stress in uremic rats. Am J Nephrol 2006; 26: 455-461.

18 Motojima M, Hosokawa A, Yamato H, Muraki T, Yoshioka T. Uremic toxins of organic anions up-regulate PAI-1 expression by induction of NF- $\mathrm{KB}$ and free radical in proximal tubular cells. Kidney Int 2003; 63: 1671-1680.

19 Owada S, Goto S, Bannai K, Hayashi H, Nishijima F, Niwa T. Indoxyl sulfate reduces superoxide scavenging activity in the kidneys of normal and uremic rats. Am J Nephrol 2008; 28: 446-454.

20 Namikoshi T, Tomita N, Satoh M, Haruna Y, Kobayashi S, Komai N, Sasaki T, Kashihara N. Olmesartan ameliorates renovascular injury and oxidative stress in Zucker obese rats enhanced by dietary protein. Am J Hypertens 2007; 10: 1085-1091.

21 Namikoshi T, Satoh M, Tomita N, Haruna Y, Kobayashi S, Komai N, Sasaki T, Kashihara N. Pioglitazone ameliorates endothelial dysfunction in obese rats with nephropathy. Biochem Biophys Res Commun 2007; 361: 835-840.

22 Tomita N, Higaki J, Kaneda Y, Yu H, Morishita R, Mikami H, Ogihara T. Hypertensive rats produced by in vivo introduction of the human renin gene. Circ Res 1993; 73: 898-905.

23 Namikoshi T, Tomita N, Satoh M, Haruna Y, Kobayashi S, Komai N, Sasaki T, Kashihara N. Pioglitazone enhances the antihypertensive and renoprotective effects of candesartan in Zucker obese rats fed a high-protein diet. Hypertens Res 2008; 31: 745-755.

24 Schrader LI, Kinzenbaw DA, Johnson AW, Faraci FM, Didion SP. IL-6 deficiency protects against angiotensin II induced endothelial dysfunction and hypertrophy. Arterioscler Thromb Vasc Biol 2007; 27: 2576-2581.

25 Hucks D, Thuraisingham RC, Raftery MJ, Yaqoob MM. Homocysteine induced impairment of nitric oxide-dependent vasorelaxation is reversible by the superoxide dismutase mimetic TEMPOL. Nephrol Dial Transplant 2004; 19: 1999-2005.

26 Dou L, Jourde-Chiche N, Faure V, Cerini C, Berland Y, Dignat-George F, Brunet P. The uremic solute indoxyl sulfate induces oxidative stress in endothelial cells. J Thromb Haemost 2007; 5: 1302-1308.

27 Shimoishi K, Anraku M, Kitamura K, Tasaki Y, Taguchi K, Hashimoto M, Fukunaga E, Maruyama T, Otagiri M. An oral adsorbent, AST-120 protects against the progression of oxidative stress by reducing the accumulation of indoxyl sulfate in the systemic circulation in renal failure. Pharm Res 2007; 24: 1283-1289.

28 Namikoshi T, Tomita N, Fujimoto S, Haruna Y, Ohzeki M, Komai N, Sasaki T, Yoshida A, Kashihara N. Isohumulones derived from hops ameliorate renal injury via an antioxidative effect in Dahl salt-sensitive rats. Hypertens Res 2007; 30: 175-184.

29 Brodsky SV, Gealekman O, Chen J, Zhang F, Togashi N, Crabtree M, Gross SS, Nasjletti A, Goligorsky MS. Prevention and reversal of premature endothelial cell senescence and vasculopathy in obesity-induced diabetes by ebselen. Circ Res 2004; 94: 377-384.

30 Fujimoto S, Satoh M, Horike H, Hatta H, Haruna Y, Kobayashi S, Namikoshi T, Arakawa S, Tomita N, Kashihara N. Olmesartan ameliorates progressive glomerular injury in subtotal nephrectomized rats through suppression of superoxide production. Hypertens Res 2008; 31: 305-313.

31 Förstermann U, Münzel T. Endothelial nitric oxide synthase in vascular disease: from marvel to menace. Circulation 2006; 113: 1708-1714.

32 Beckman JS, Koppenol WH. Nitric oxide, superoxide, and peroxynitrite: the good, the bad, and ugly. Am J Physiol 1996; 271: C1424-C1437.

33 Dou L, Bertrand E, Cerini C, Faure V, Sampol J, Vanholder R, Berland Y, Brunet P. The uremic solutes $\mathrm{p}$-cresol and indoxyl sulfate inhibit endothelial proliferation and wound repair. Kidney Int 2004; 65: 442-451.

34 Faure V, Dou L, Sabatier F, Cerini C, Sampol J, Berland Y, Brunet P, Dignat-George F. Elevation of circulating endothelial microparticles in patients with chronic renal failure. J Thromb Haemost 2006; 4: 566-573.

35 Taki K, Tsuruta Y, Niwa T. Indoxyl sulphate and atherosclerotic risk factors in hemodialysis patients. Am J Nephrol 2007; 27: 30-35.

36 Wilcox CS. Oxidative stress and nitric oxide deficiency in the kidney: a critical link to hypertension? Am J Physiol Regul Integr Comp Physiol 2005; 289: 913-935. 